\title{
Light intensity enhancement by diffracting structures in solar cells
}

\author{
I. Tobías, ${ }^{\text {a) }}$ A. Luque, and A. Martí \\ Instituto de Energía Solar, Universidad Politécnica de Madrid, Madrid 28040, Spain
}

(Received 7 March 2008; accepted 27 May 2008; published online 1 August 2008)

\begin{abstract}
A simplified three-dimensional study is presented of the light confinement, that is, of the enhancement of the Poynting vector of the electromagnetic radiation of the light inside a solar cell absorbing the light weakly when diffracting structures are used. The model is based on the theory of periodic radiation arrays and is easily applied to one- and two-dimensional diffraction gratings. Realistically wide illumination bundles are considered. The extended nature of illumination severely limits the enhancement capabilities of diffraction structures. Results are compared to those of the more widely used Lambertian light confinement. (C) 2008 American Institute of Physics.
\end{abstract}

[DOI: 10.1063/1.2960586]

\section{INTRODUCTION}

Some solar cells have a weak light absorption because they are thin or, at wavelengths close to the absorption edge, because of the indirect nature of their bandgap, as in the case of silicon devices. Furthermore, certain solar cell types and, in particular, the intermediate band solar cells ${ }^{1}$ (IBSCs) - in which electron-hole pairs are created out of sub-band-gap photons by means of quantum dots ${ }^{2}$ (QDs) or by alloys of different types ${ }^{3-5}$-absorb weakly the sub-band-gap photons because of the low density of absorbing centers (below $10^{17} \mathrm{~cm}^{-3}$ in the QD-IBSC). It is to be noted that most probably QD-IBSC will not be practical unless they can utilize a good scheme of light-absorption intensification.

To enhance the absorption we can randomize the light direction inside the semiconductor that forms the solar cell ${ }^{6,7}$ hindering its exit from the semiconductor thanks to the higher density of modes inside it, that is proportional to the square $n^{2}$ of its index of refraction: rays that are not coupled to outside modes cannot escape by the total internal reflection. This is the so-called Lambertian confinement. An alternative to it is the enhancement of the light intensity based on diffraction. The use of diffraction structures was proposed for solar cells in 1989 (Ref. 8) although the procedures to analyze the problem numerically were developed earlier., ${ }^{9,10}$

The intensity enhancement produced by a diffraction grating is often interpreted in terms of an increase in the path of the rays inside the semiconductor and referred to as light confinement. It can be also looked at as an increase in the electromagnetic field when it experiences a change of direction, thus enhancing the absorption of the light.

The change of direction caused by diffraction on a plane wave produces an increase in the strength of the Poynting vector of the deflected beam. In effect, we represent in Fig. 1 an electromagnetic plane wave with the wave vector in the direction of the arrow (direction unity vector $\boldsymbol{u}_{i}$ ) which is deflected (direction unity vector $\boldsymbol{u}_{0}$ ) when traversing a surface $\Sigma$. The angles with the normal $\left(\boldsymbol{u}_{z}\right)$ to the surface element $d \Sigma$ of the incident wave vector and the deflected wave vector are $\theta_{i}$ and $\theta_{0}$, respectively. The Poynting vectors of

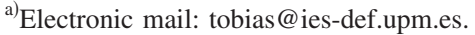

incident and deflected waves $\boldsymbol{S}_{i}$ and $\boldsymbol{S}_{0}$ (modules $S_{i}$ and $S_{0}$ ) are in the direction of the wave vectors. If all the power in the incident wave is transmitted to the deflected wave, the conservation of energy states that $S_{i} \cos \theta_{i} d \Sigma=S_{0} \cos \theta_{0} d \Sigma$ so that

$$
S_{0}=S_{i} \frac{\cos \theta_{i}}{\cos \theta_{0}} .
$$

If $\theta_{0}$ approaches $90^{\circ}$, the deflected wave Poynting vector tends to infinity. This simple result introduces an exciting concept: if we are able to deflect the rays horizontally the Pointing vector inside the semiconductor will be infinite and the absorption will be very much increased. Diffraction seems a way to achieving this.

Most of the work done so far has been limited to diffraction gratings and their analysis is generally two dimensional (2D), ${ }^{11}$ that is, confined to light propagating in the plane normal to the diffraction grating lines, because the exact three-dimensional (3D) treatment involves considerable complexity. $^{12}$

In this paper we present an approximate $3 \mathrm{D}$ model based on the techniques widely used in electromagnetism to study arrays of radiators. ${ }^{9,13}$ To this purpose we shall obtain the diffraction directions with a general formalism applicable to one-dimensional (1D), 2D, and 3D arrays of diffraction centers when they are periodic. Then we analyze the conse-

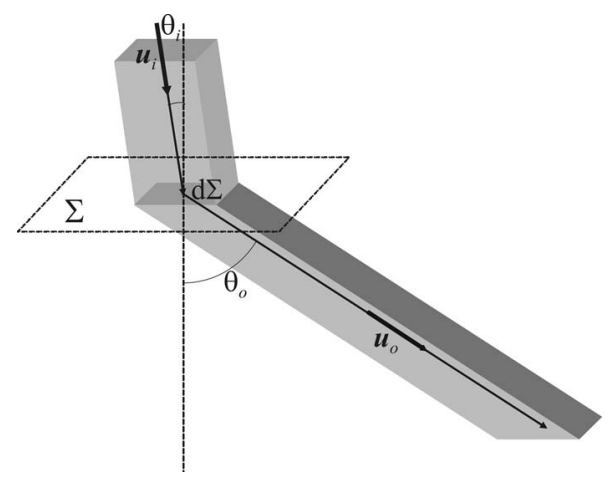

FIG. 1. A plane wave incident on a surface $\Sigma$ with an angle $\theta_{i}$ is deflected when traversing it to an angle $\theta_{0}$. 
quences of the angle-extended illumination of the solar cells, which are excited by a bundle of plane waves, generally incoherent, and not by a single plane wave. As we shall see this greatly reduces the Poynting-vector-increasing potential of this concept. Finally, we calculate the confinement enhancement that we can expect from these structures and discuss how this compares to the Lambertian confinement proposed by Yablonovitch and Cody. ${ }^{7}$

\section{CONSTRUCTIVE INTERFERENCE DIRECTIONS}

\section{A. Diffraction by a single object}

Let us assume an illumination light consisting in a plane wave represented by the real or imaginary part of the complex disturbance,

$$
\boldsymbol{E}_{i}=E_{0} \boldsymbol{u}_{x} \exp \left(i \boldsymbol{k}_{i} \cdot \boldsymbol{r}-i \omega t\right) \quad \forall \quad \omega=k c / n=k / \sqrt{\varepsilon \mu},
$$

$\boldsymbol{E}_{i}$ is the electric field vector, $E_{0}$ its complex amplitude, and $\boldsymbol{k}_{i}$ the wavevector, whose amplitude $k$ is related to the angular frequency $\omega$ through the light speed in the medium $c / n$. Coordinate axes are taken so that the $x$-axis, with unity vector $\boldsymbol{u}_{x}$, contains the polarization vector.

An object is placed at the origin of coordinates, small as compared to the distance of observation $r$. By positioning this object, the illuminating field can be considered as unchanged but a diffracted spherical wave will appear that neglecting the near field behavior can be represented by ${ }^{8,13}$

$$
\boldsymbol{E}_{d}(\boldsymbol{r})=\frac{E_{0} \boldsymbol{\Lambda}(\boldsymbol{r} / r)}{r} \exp i(k r-\omega t),
$$

$\boldsymbol{\Lambda}(\boldsymbol{r} / r)$ is a complex vector function that gives the dependence of the diffracted field on the observation direction. In the rest of the paper the factor $\exp (-i \omega t)$ appearing in any field term will be omitted.

For large $r$, this spherical wave behaves locally as a transversal plane wave with wave vector $\boldsymbol{k}=k \boldsymbol{u}_{r}$, where $\boldsymbol{u}_{r}$ $=\boldsymbol{r} / \boldsymbol{r}$ is a unit vector in the direction of $\boldsymbol{r}$. The following relationships are fulfilled:

$$
\begin{aligned}
& \boldsymbol{E} \cdot \boldsymbol{H}=\boldsymbol{E} \cdot \boldsymbol{k}=\boldsymbol{H} \cdot \boldsymbol{k}=0, \\
& E=\sqrt{\mu / \varepsilon} H=\eta H .
\end{aligned}
$$

Let us consider the effect of displacing the diffraction center to the position $\boldsymbol{d}$. The diffracted field is

$$
\boldsymbol{E}_{d}(\boldsymbol{r})=\frac{E_{0} \boldsymbol{\Lambda}[(\boldsymbol{r}-\boldsymbol{d}) /|\boldsymbol{r}-\boldsymbol{d}|]}{|\boldsymbol{r}-\boldsymbol{d}|} \exp (i k|\boldsymbol{r}-\boldsymbol{d}|) \exp \left(i \boldsymbol{k}_{i} \cdot \boldsymbol{d}\right)
$$

The factor $\exp \left(i \boldsymbol{k}_{i} \cdot \boldsymbol{d}\right)$ accounts for the phase difference of the illuminating plane wave when the center is displaced from its original position.

We assume now that $d$ is small enough as to keep the amplitude vector $E_{0} \boldsymbol{\Lambda}(\boldsymbol{r} / r) / r$ unchanged, but not small with respect to wavelength so that the phase factor is changed. First order approximation in $d$ gives

$$
\boldsymbol{E}_{d}(\boldsymbol{r}) \cong \frac{E_{0} \boldsymbol{\Lambda}\left(\boldsymbol{u}_{r}\right)}{r} \exp (i k r) \exp \left[-i\left(k \boldsymbol{u}_{r}-\boldsymbol{k}_{i}\right) \cdot \boldsymbol{d}\right]
$$

It is very common that the diffraction centers are at the interface of two different media. In each one of them the wavelengths are different and obtained with the formulas in Eq. (2) using the different index of refraction of each medium. Furthermore, refractions and reflections take place, producing complex field configurations for both the illuminating plane wave and for the scattered spherical wave. We assume for the moment that the scattering centers are inside a given semiconductor.

\section{B. Diffraction by arrays}

\section{3D array of diffractive centers}

Let us now consider an array of diffractive centers located at positions $\boldsymbol{a}=m_{1} \boldsymbol{a}_{1}+m_{2} \boldsymbol{a}_{2}+m_{3} \boldsymbol{a}_{3}$, with $m_{k}(k=1,2,3)$ an integer. This forms a periodical lattice whose reciprocal lattice is $\boldsymbol{B}=p_{1} \boldsymbol{B}_{1}+p_{2} \boldsymbol{B}_{2}+p_{3} \boldsymbol{B}_{3}$ (for $p_{k}$ integer), where $\boldsymbol{B}_{1}$ $=\left(\boldsymbol{a}_{2} \times \boldsymbol{a}_{3}\right) / \boldsymbol{a}_{1}\left(\boldsymbol{a}_{2} \times \boldsymbol{a}_{3}\right)$ and $\boldsymbol{B}_{2}$ and $\boldsymbol{B}_{3}$ are obtained in the same way by circular permutation of indices. These vectors have the property $\boldsymbol{a}_{i} \cdot \boldsymbol{B}_{j}=\boldsymbol{\delta}_{i, j}$. All the centers are supposed to be found inside a homogeneous medium to which all calculations are referred.

We further assume that the diffraction centers in the array are illuminated only by the incident wave and not by light scattered by the other centers. In this case, and assuming also that the observation point is far away from all the diffractive centers,

$$
\begin{aligned}
\boldsymbol{E}_{d}(\boldsymbol{r}) \cong & \frac{E_{0} \boldsymbol{\Lambda}\left(\boldsymbol{u}_{r}\right)}{r} \exp (i k r) \\
& \times \sum_{m 1, m 2, m 3} \exp \left[-i\left(k \boldsymbol{u}_{r}-\boldsymbol{k}_{i}\right) \cdot \boldsymbol{a}_{m 1, m 2, m 3}\right] .
\end{aligned}
$$

The summation is of a set of complex numbers of module unity and random phase, thus the sum is zero except when

$$
\boldsymbol{u}_{r}=\boldsymbol{B} \lambda+\boldsymbol{k}_{i} / k=\boldsymbol{B} \lambda+\boldsymbol{u}_{i} .
$$

When Eq. (8) applies, the phase of the terms is $-2 \pi\left(m_{1} p_{1}\right.$ $\left.+m_{2} p_{2}+m_{3} p_{3}\right)$, that is, an integer number of $2 \pi$ for any value of the integers $m_{1}, m_{2}$, and $m_{3}$, and therefore the exponentials become unity and their sum is $N$, the number of diffractive centers.

The zero sum out of the condition in Eq. (8) is fulfilled exactly for an infinite number of diffracting centers. For a finite number of them, it holds approximately, and approaches $N$ when the equality in this equation is only approximate.

Let us stress that $\boldsymbol{u}_{r}=\boldsymbol{B} \boldsymbol{\lambda}+\boldsymbol{u}_{i}$ must be a unity vector to represent a diffraction direction. There is a set of candidate nodes of the reciprocal lattice normalized to $1 / \lambda$, taking as origin the unity vector of the illuminating plane wave. However, only those situated on the unity-radius sphere (or perhaps, in its proximity, if the number of diffraction centers is not very large) are direction vectors of the diffracted beams.

In the case of a very large (infinite) number of diffraction centers only the constructive interference directions occur and therefore the incident wave is diffracted into a certain number of plane waves, the so-called diffraction orders. Despite the weakness of the spherical wave scattered by a single 
center, its ensemble can lead to a substantial part of the illuminating power density to be transferred to the scattered waves.

Note that changes of the incoming beam direction displace the reciprocal lattice and may change the nodes located on the unity-radius sphere. It may happen that under certain (or all) illumination directions, no diffraction direction is found beside the illumination direction.

Effectively, one of the points of the reciprocal lattice is the point $(0,0,0)$. This means that the direction $\boldsymbol{u}_{i}$ of the incident light is always a possible diffraction direction. The reason is that, by Huygens' principle, the illuminating wave makes all the scattering points to emit with a phase able to reconstitute the incident flat wave.

If the conditions permitting the approximations in Eq. (6) are not fulfilled, that is, if the distance from the scattering centers to the point of observation is not the same, or if the direction from which the scattering centers are seen at the observation point is different, only a few of the centers will contribute to the field at the observation point and therefore the electromagnetic field does not go to zero out of the directions dictated by Eq. (8). In this case there are a number of radiation lobes around the allowed directions; these lobes are not sharp and there may be secondary ones as well. This situation can be found in some solar cells in which the diffraction centers are very close to the active part of the cell, for instance, in the front face of a III-V solar cell. However, if they are at the back of the substrate the approximations leading to Eq. (6) are rather accurate.

\section{2D array of diffractive centers and diffraction gratings}

We can describe a 2D array of diffraction centers as a degenerate $3 \mathrm{D}$ array, such that the plane of scattering centers is at the $x y$-plane and successive parallel planes are infinitely separated. In other words, $\boldsymbol{a}_{3}=\Omega \boldsymbol{u}_{z}$ with $\Omega \rightarrow \infty$. If this is the case,

$$
\begin{aligned}
& \boldsymbol{B}_{1}=\frac{\boldsymbol{a}_{2} \times \boldsymbol{u}_{z}}{\boldsymbol{a}_{1}\left(\boldsymbol{a}_{2} \times \boldsymbol{u}_{z}\right)}, \\
& \boldsymbol{B}_{2}=\frac{\boldsymbol{u}_{z} \times \boldsymbol{a}_{1}}{\boldsymbol{a}_{2}\left(\boldsymbol{u}_{z} \times \boldsymbol{a}_{1}\right)}, \\
& \boldsymbol{B}_{3}=\frac{\boldsymbol{a}_{1} \times \boldsymbol{a}_{2}}{\Omega \boldsymbol{u}_{z}\left(a_{2} \times \boldsymbol{a}_{1}\right)}=\boldsymbol{\varpi} \boldsymbol{u}_{z},
\end{aligned}
$$

with $\varpi \rightarrow 0$. In this situation, the reciprocal lattice points form a set parallel lines in the $z$ direction as represented in Fig. 2 (once $\boldsymbol{B}_{1}$ and $\boldsymbol{B}_{2}$ are multiplied by $\lambda$ ). According to Eq. (8) the directions of constructive interference are those of intersection of these lines with the sphere of radius unity.

In the case of an array of diffracting lines (a diffraction grating) located in the $x y$-plane, they can be represented as a $3 \mathrm{D}$ array of points such that $\boldsymbol{a}_{1}=\varepsilon \boldsymbol{u}_{1}$ with $\varepsilon \rightarrow 0, \boldsymbol{a}_{2}=a \boldsymbol{u}_{2}$, and $\boldsymbol{a}_{3}=\Omega \boldsymbol{u}_{z} \cdot \boldsymbol{u}_{1}$ and $\boldsymbol{u}_{2}$ are a couple of orthogonal vectors located in the $x y$-plane but possibly rotated with respect to $\boldsymbol{u}_{x}$ and $\boldsymbol{u}_{y}$ (remember that the electric polarization is along $\boldsymbol{u}_{x}$ ). In consequence,

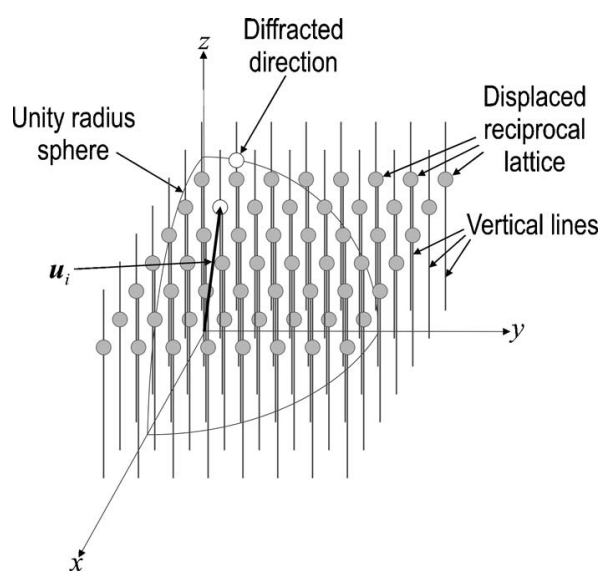

FIG. 2. The reciprocal lattice of a $2 \mathrm{D}$ array of diffracting centers times $\lambda$, once displaced by $\boldsymbol{u}_{i}$. The intersection of the vertical lines and the surface of the sphere of radius unity represent valid diffraction directions.

$$
\begin{aligned}
& \boldsymbol{B}_{1}=\frac{\boldsymbol{u}_{2} \times \boldsymbol{u}_{z}}{\varepsilon \boldsymbol{u}_{1}\left(\boldsymbol{u}_{2} \times \boldsymbol{u}_{z}\right)}=\frac{\boldsymbol{u}_{1}}{\varepsilon}, \\
& \boldsymbol{B}_{2}=\frac{\boldsymbol{u}_{z} \times \boldsymbol{u}_{1}}{a \boldsymbol{u}_{2}\left(\boldsymbol{u}_{z} \times \boldsymbol{u}_{1}\right)}=\frac{\boldsymbol{u}_{2}}{a}, \\
& \boldsymbol{B}_{3}=\frac{\varepsilon a \boldsymbol{u}_{1} \times \boldsymbol{u}_{2}}{\Omega \varepsilon a \boldsymbol{u}_{z}\left(\boldsymbol{u}_{1} \times \boldsymbol{u}_{2}\right)}=\varpi \boldsymbol{u}_{z} .
\end{aligned}
$$

This means that $\boldsymbol{B}_{1} \lambda$ is very large and all the diffraction directions associated with $p_{1} \neq 0$ are outside the unity-radius sphere and must be discarded. Thus the only nodes of the reciprocal lattice to be taken into account are in the direction $\boldsymbol{u}_{2}$, normal to the diffractive lines (that run in the direction $\boldsymbol{u}_{1}$ ), separated $1 / a$. As seen in Fig. 3, from each of these points emerges a vertical line formed by the successive points along vector $\boldsymbol{B}_{3}$, forming a continuous as in the $2 \mathrm{D}$ case. To obtain the scattering directions we must locate the reciprocal lattice multiplied by $\lambda$ at the extreme of the unity vector along the illumination direction.

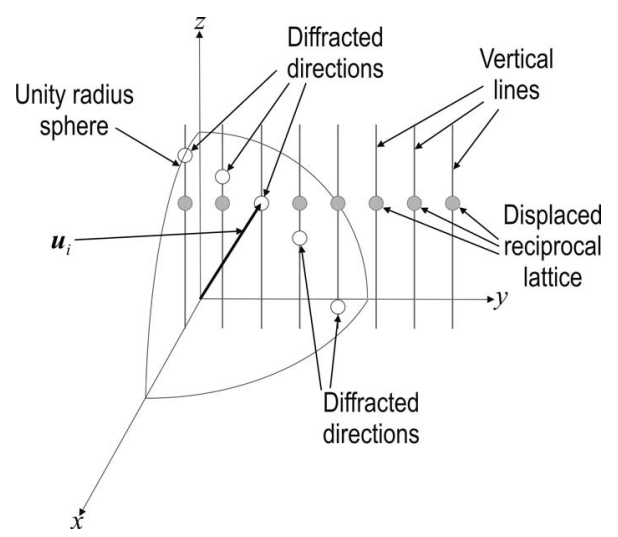

FIG. 3. The reciprocal lattice of an array of diffractive lines times $\lambda$, once displaced by $\boldsymbol{u}_{i}$. The intersection of the vertical lines and the surface of the sphere of radius unity represent valid diffraction directions. In this drawing the array of reciprocal lattice nodes runs parallel to the $y$ axis implying that the diffraction lines are in the $x$ direction but any other horizontal direction is equally valid. 


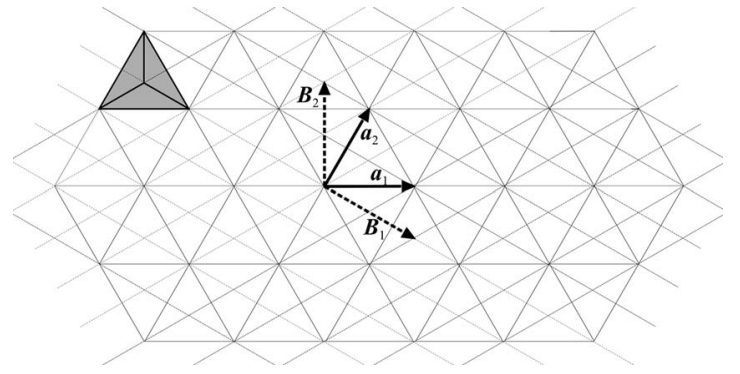

FIG. 4. The diffraction center lattice (solid lines) and the reciprocal lattice (dashed lines) of a set of pyramids of triangular base engraved on the surface of a solar cell.

It is clear by this construction, in the two cases discussed, that allowed diffraction is produced not only with a normal $(z)$ component in the direction of the illuminating wave (transmission) but also in the opposite direction (reflection).

\section{The effect of an interface}

An important situation for solar cells is the one in which the scattering centers are engraved on a surface. In the case of the front surface, interesting diffraction takes place in transmission to the substrate, so that the diffracted waves propagate in a medium different to the one illumination is coming from. For the back surface, on the contrary, the main interest lies on diffraction on reflection, so that it occurs in the same medium as the illumination since the change of medium takes place at the front face.

In the case of diffraction with change of medium, Eqs. (5)-(7) remain valid, but now the wave is scattered in a medium with index of refraction $n$, while illumination comes from a medium with refraction index of 1 . It can thus be stated that $c / \omega=k_{i}=k / n$, and Eq. (8) transforms into

$$
\boldsymbol{u}_{r}=\boldsymbol{B} \boldsymbol{\lambda}+\boldsymbol{k}_{i} / k=\boldsymbol{B} \boldsymbol{\lambda}+\boldsymbol{u}_{i} / n .
$$

When the hemisphere of illumination is the same as the hemisphere of interest for scattering, the treatment in the preceding sections is valid, using of course the relationship between wave vector and angular frequency corresponding to the index of refraction of the medium involved. If the index of refraction of the incident medium is different from 1 , then $n$ must be replaced by the ratio of $n$ 's. Of course, the relationship between wave vector and angular frequency for the incident medium has to be modified as well.

\section{Angle-extended illumination}

\section{Scattering directions in $2 D$ arrays}

To develop our discussion, let us consider a 2D array of scattering centers consisting of triangular-base pyramids. In Fig. 4 one of these pyramids and the lattice of scattering centers are presented, together with the reciprocal lattice. The unity vectors of the real space lattice are $\boldsymbol{a}_{1}=(1,0,0) a$ and $\boldsymbol{a}_{2}=(1 / 2, \sqrt{3} / 2,0) a$. The reciprocal lattice unity vectors are $\boldsymbol{B}_{1}=(\sqrt{ } 3 / 2,-1 / 2,0)(2 / a \sqrt{ } 3)$ and $\boldsymbol{B}_{2}=(0,1,0)(2 / a \sqrt{ } 3)$.

Frequently a solar cell receives the light in a cone with semiangle $\Theta_{\text {sun }} \cong 0.2670^{\circ}$ that subtends the sun disk. More common for high efficiency cells is to be illuminated by a
TABLE I. Some data of extended illumination bundles.

\begin{tabular}{lccc}
\hline \hline & $\begin{array}{c}\text { Direct } \\
\text { sun }\end{array}$ & $\begin{array}{c}\text { Ideal } \\
\text { concentrator }\end{array}$ & $\begin{array}{c}1^{\circ} \\
\text { concentrator }\end{array}$ \\
\hline Subtended disk semiangle $\left(^{\circ}\right)$ & 0.267 & 0.267 & 1 \\
Concentration (no secondary) & 1 & 500 & 500 \\
Concentration $n_{\text {optics }}=1.5$ & & 1125 & 1125 \\
Output angle $\Theta_{\text {out }}\left({ }^{\circ}\right)$ & 0.267 & 5.981 & 22.970 \\
Index of refraction $n$ & $\sqrt{ }(11$ & $\sqrt{ } 11$ & $\sqrt{11}$ \\
Radius of projected bundle $\delta$ & 0.0014 & 0.0314 & 0.1177 \\
Refracted output angle $\Theta_{x}\left(^{\circ}\right)$ & 0.081 & 1.800 & 6.757 \\
\hline \hline
\end{tabular}

concentrator whose output semiangle is $\Theta_{\text {out }}$. For an ideal concentrator, the concentration factor is ${ }^{14} C$ $=n_{\text {optics }}^{2} \sin ^{2} \Theta_{\text {out }} / \sin ^{2} \Theta_{\text {sun }}$, where $n_{\text {optics }}$ is the refraction index of the optical material used to fabricate surrounding the cell. An ideal concentrator with no secondary optics and $C$ $=500$ will have $\Theta_{\text {out }}=5.981^{\circ}$. An ideal concentrator with the same output angle to which a secondary with $n_{\text {optics }}=1.5$ has been added will achieve $C=1125$, that discounting the optical losses and assuming perfect tracking might lead to an irradiance concentration of 1000. A third case of interest is the use of a concentrator to collect all the rays from a cone of semiangle of $1^{\circ}$ around the sun. This allows for manufacturing inaccuracies in the concentrator and in the sun aiming. We present in Table I a collection of useful data concerning the three illumination bundles mentioned before. Last row gives their semiangles once they have entered the semiconductor material through a flat interface.

As indicated in Eq. (1), the more inclined the $k$-vector is, the higher the Poynting vector of the diffracted wave. As explained in Sec. II B 2, the diffraction directions under normal illumination are the vertical projections of the reciprocal lattice times $\lambda$ on the unity-radius sphere. For this normal illumination, if we make $B_{1} \lambda=B_{2} \lambda=1$, these projections are on the circumference of the circle of radius unity drawn in Fig. 5, the diffraction directions are horizontal and the Poynting vector is infinite. From another point of view, this means that the path of the diffracted ray will never leave the cell and the illumination light will be totally absorbed. As indicated in the figure, in this way we have six horizontal diffraction directions.

However, the light is coming in a cone of semiangle $\Theta_{\text {out }}$. The unity vectors of the illumination plane waves have projections on the $x y$-plane given by $p \equiv \boldsymbol{u}_{i} \cdot \boldsymbol{u}_{x}, q \equiv \boldsymbol{u}_{i} \cdot \boldsymbol{u}_{y}$,

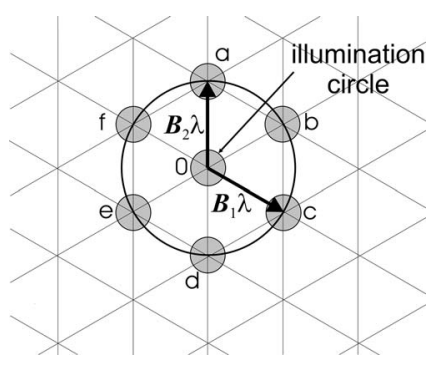

FIG. 5. Reciprocal lattice of a $2 \mathrm{D}$ array of diffractive centers times $\lambda$, unity-radius circle, illumination circle, and diffracted circles. Their centers are on the reciprocal arrays nodes located on the circumference with unity radius. 


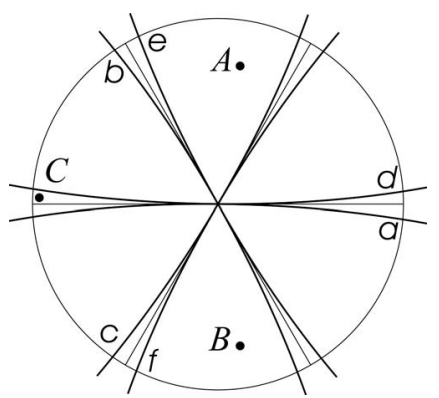

FIG. 6. The 0 circle of Fig. 5 and the circles $a-f$ translated into it with the arc of unity-radius circle associated with them. The arcs are labeled with the same letters as the circles $a-f$ to which they cross in Fig. 5. $A, B$, and $C$ are illumination directions.

the direction cosines. The distance of these projections to the origin is $\sqrt{p^{2}+q^{2}}=\sqrt{1-\cos ^{2} \theta}=\sin \theta$, with $\theta$ the angle formed with the vertical $\left(\boldsymbol{u}_{z}\right)$ direction. Therefore all directions have $\theta \leq \Theta_{\text {out }}$.

The reciprocal lattice node projections on the $x y$ plane, for rays within the illumination cone, are displaced from their original positions by vectors situated inside circles of radius,

$$
\delta=\sin \Theta_{\text {out }} / n \equiv \sin \Theta_{x} .
$$

The $n$ divisor is a direct application of Snell's law, for illumination light inside the semiconductor (back surface diffracting lattice), or is a direct application of Eq. (11) for illumination light from outside (front surface diffracting lattice). In the absence of concentrator $\Theta_{\text {out }}=\Theta_{\text {sun }}$.

The condition $B_{1} \lambda=B_{2} \lambda=1$ corresponds in this case to $a / \lambda=2 / \sqrt{3}$. This information (remembering that $\lambda$ is the wavelength inside the semiconductor) allows calculating an initial proposal for the spacing of the pyramids.

A number of circles (labeled $0, a, b, c, d, e, f$ ) around the nodes of the reciprocal lattice are represented in Fig. 5, according to the discussion above. Due to the procedure for sizing the reciprocal lattice the small circles $a-f$ are partly outside the unity-radius circle and partly inside. The reciprocal lattice points projected in the external part of the circles $a-f$ do not lead to any diffraction direction. This means that for certain illumination directions not all the six "horizontal" diffraction directions will actually take place. On the other hand only the light diffracted on the unity circle radius circumference is really horizontal. Other direction in the horizontal lobes are actually very inclined but not horizontal.

In Fig. 6 we draw the circle 0 and translate onto it the six circles $a-f$, included in this translation the fraction of unity circle inside them. This arc segment is labeled with the lower case letter corresponding to the circle. The drawing helps understanding the diffraction directions associated with each illumination direction. For instance, the illumination direction represented by point $A$ (in the 0 circle) has diffraction in directions belonging to circles $0, d, c$, and $e$, but not in circles $a, f$, or $b$. On the contrary, for direction $B$ in the 0 circle, diffraction directions are only possible in circles $0, a$, $b$, and $f$. For point $C$ diffraction directions are located in

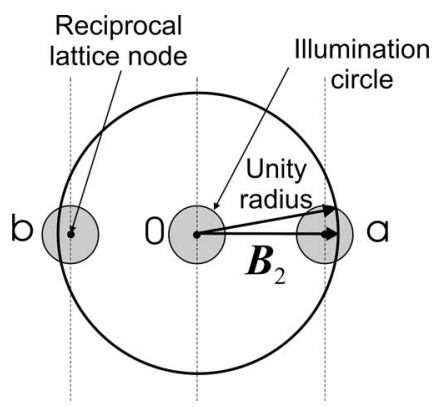

FIG. 7. The circle of unity radius and the illumination (0) and scattering $(a, b)$ circles. The scattering circle centers are located at the nodes of the reciprocal lattice but not on the unity radius.

circles $0, b$, and $c$. For vertical illumination there is diffraction in all the lobes $0, a, b, c, d, e, f$, as discussed before.

Sizing the reciprocal lattice so that the centers of the circles $a-f$ are outside the unity-radius circle would leave a region in the center of the circle of Fig. 6 where diffraction would not be coupled to any horizontal lobe.

The preceding scheme can be used with small modifications for a 2D array of square base pyramids.

\section{Scattering directions in diffraction gratings}

As discussed in Sec. II B 2, the reciprocal lattice in this case involves a linear set of nodes and $\boldsymbol{B}_{2}$ is a vector of module $1 / a$ normal to the diffraction lines. When the light comes from a cone of rays, represented by the circle 0 in Fig. 7 , it will be diffracted along directions into the circles $a$ and $b$, and also along the directions in the circle 0 .

For this reason, too, the centers of circles $a$ and $b$, which are on the reciprocal lattice nodes, should be located on the circumference of the unity circle. However, for analysis purposes, we admit other possible situations so that the centers of circles $a$ and $b$ are at abscissas $h$ and $-h$, respectively $(B \lambda= \pm h)$. In Fig. 8 the horizontal lobe circles have been translated and superimposed to the 0 circle, bringing with them the arc of unity circumference crossing them (again labeled $a$ and $b$ ).

In this way, we see that the illumination in the direction $A$ will be diffracted toward directions in circles 0 or $b$ but not in circle $a$, while illumination in direction $B$ will be dif-

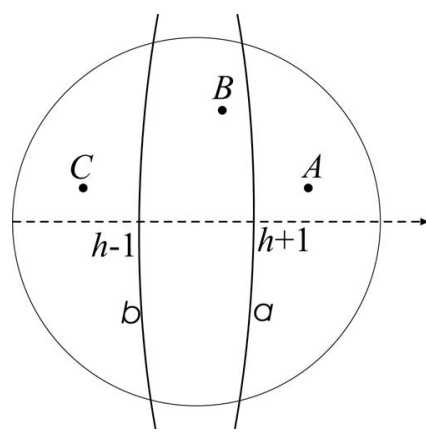

FIG. 8. The 0 circle of Fig. 7 and the circles $a$ and $b$ translated onto it with the arc of unity-radius circle associated with them. The arcs are labeled with the same letters as the which they cross in Fig. 7. $A, B$, and $C$ are illumination directions. 


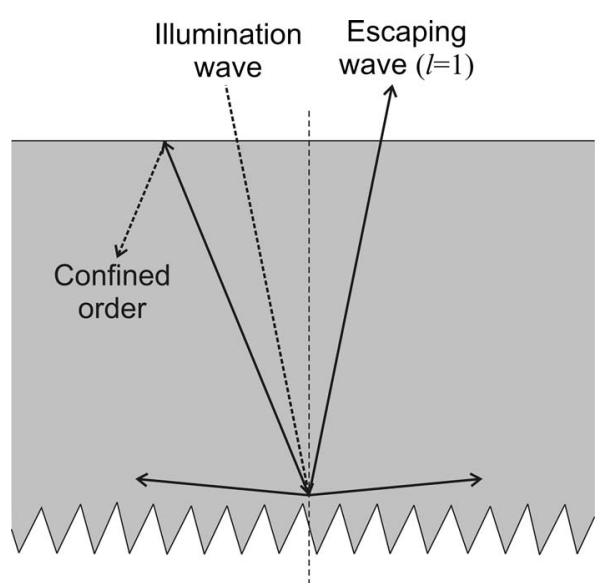

FIG. 9. Solar cell with a reflective diffraction grating at the back where an illumination wave is diffracted. The specularly reflected wave escapes through the front face, while laterally scattered waves suffer total internal reflection there and impinge on the diffraction grating, exciting again the upward-traveling modes.

fracted along directions in all the circles: $0, a$, and $b$. Illumination in direction $C$ will be diffracted toward directions in circles 0 or $a$, but not in circle $b$. With the construction in Fig. 8 illumination in the direction $B$ would be deflected on both horizontal lobes (plus the vertical lobe), but if $h$ exceeds the unity circle radius, region $B$ will disappear and some incoming directions will not be diffracted into any horizontal lobe. However, as far as regions $A$ and $C$ exist, rays will be more inclined, producing a higher increase in the Poynting vector. Further analysis will be performed in Sec. III.

\section{POWER CALCULATIONS}

\section{A. Confinement of diffracted modes}

Let us consider a diffraction grating engraved on the back face of a solar cell, as represented in Fig. 9. Light impinges on the grating, that lies on the $x y$-plane from inside the semiconductor. In line with the subject under study of very weakly absorbing cells, the semiconductor is assumed to be transparent. Perfect reflection is assumed, too. Equation (8) for planar 1D or 2D reflection gratings is written for the tangential (projected on the $x y$-plane) components of the incident $\left(\boldsymbol{u}_{i t}\right)$ and diffracted $\left(\boldsymbol{u}_{r t}\right)$ direction vectors as

$$
\boldsymbol{u}_{r t}=\boldsymbol{B} \lambda+\boldsymbol{k}_{i t} / k=m_{1} \boldsymbol{B}_{1} \lambda+m_{2} \boldsymbol{B}_{2} \lambda+\boldsymbol{u}_{i t},
$$

where only $m_{1}=0$ makes sense for a $1 \mathrm{D}$ grating. The propagating waves are those for which $\left|\boldsymbol{u}_{r t}\right| \leq 1$.

In the structure depicted in Fig. 9, the reflected waves travel upward up to the front face; another reflection takes place there that leaves tangential direction vectors unchanged, so that when they reach the back face again the set of scattered modes is left the same. In effect, let us consider that the mode given by the numbers $m_{1}$ and $m_{2}$ impinges on the grating after reflection. The scattered waves fulfill

$$
\begin{aligned}
\boldsymbol{u}_{i t}^{\prime} & \equiv \boldsymbol{u}_{r t\left(m_{1}, m_{2}\right)} \Rightarrow \boldsymbol{u}_{r t\left(m_{1}^{\prime}, m_{2}^{\prime}\right)}^{\prime}=\boldsymbol{u}_{i t}^{\prime}+m_{1}^{\prime} \boldsymbol{B}_{1} \lambda+m_{2}^{\prime} \boldsymbol{B}_{2} \lambda \\
& =\boldsymbol{u}_{i t}+\left(m_{1}+m_{1}^{\prime}\right) \boldsymbol{B}_{1} \lambda+\left(m_{2}+m_{2}^{\prime}\right) \boldsymbol{B}_{2} \lambda=\boldsymbol{u}_{r t\left(m_{1}+m_{1}^{\prime}, m_{2}+m_{2}^{\prime}\right)} .
\end{aligned}
$$

It is always possible to select the indices $m_{1}^{\prime}$ and $m_{2}^{\prime}$ (positive and negative) as to fill all the reciprocal lattice vectors situated inside the circle of radius unity that were present in the scattered directions corresponding to the incident wave.

Let $N_{\text {max }}$ be the number of reflected propagating modes. In the following, these will be labeled with a single integer $1 \leq l \leq N_{\max }$ corresponding to an allowed combination of $m_{1}$ and $m_{2}$, where $l=1$ corresponds to the nondiffracted mode $\boldsymbol{u}_{r t}=\boldsymbol{u}_{i t}\left(m_{1}=m_{2}=0\right)$, which is always present. Besides, the polarization state denoted by $\mu$ can take on two values, $s$ and $p$, depending on the magnetic or the electric vector being contained in the plane of incidence.

Let $P_{l ; \mu}$ be the power flux in the direction normal to the plane of the grating (that we assume to be the $x y$-plane) carried by a plane wave (moving upward or downward, i.e., incident or reflected) with tangential direction vector $\boldsymbol{u}_{r t(l)}$ and $\mu$ polarization. $P_{(l ; \mu)}$ is the projection, in absolute value, of the time averaged Poynting vector $\langle\boldsymbol{S}\rangle$ along the normal to the plane of the grating $\boldsymbol{u}_{z}$,

$$
P_{(l ; \mu)} \equiv\left|\left\langle\boldsymbol{S}_{(l ; \mu)}\right\rangle \cdot \boldsymbol{u}_{z}\right|=\left|\left\langle S_{(l ; \mu)}\right\rangle \cos \theta\right| \text {. }
$$

If a plane wave with $\boldsymbol{u}_{r t(l)}$ impinges on the grating, all modes are reflected. By $\eta_{(k ; \mu) \rightarrow(l ; \nu)}$ it is denoted the (absolute) diffraction efficiency from the mode with label $k$ and polarization $\mu$ into mode with label $l$ with polarization $\nu$, i.e., the ratio between the power fluxes,

$$
\eta_{(k ; \mu) \rightarrow(l ; \nu)} \equiv\left|\frac{P_{(l ; \nu)}}{P_{(k ; \mu)}}\right| .
$$

When only the $k$-labeled, $\mu$-polarized mode is incident. The total reflected power can be expressed as the sum of the power carried away by each mode as shown in Eq. (A3) in the Appendix. For a perfect reflector grating such that no power is allowed to escape, energy conservation forces diffraction efficiencies to fulfill

$$
\sum_{\nu=s, p} \sum_{l=1}^{N_{\max }} \eta_{(k ; \mu) \rightarrow(l ; \nu)}=1 .
$$

On the other hand, reciprocity implies that the same efficiency values would apply if the wavevector directions were reversed $^{15}$ (that is, if $\boldsymbol{k}$ values were substituted by $\boldsymbol{-} \boldsymbol{k}$ and polarization states interchanged). There is a one-to-one relationship between the modes present in both situations, original and reversed. By labeling them with the same indices, and denoting reversed magnitudes with a prime, we have

$$
\eta_{(k ; \mu) \rightarrow(l ; \nu)}^{\prime} \equiv\left|\frac{P_{(l ; \nu)}^{\prime}}{P_{(k ; \mu)}^{\prime}}\right|=\eta_{(l ; \nu) \rightarrow(k ; \mu)} .
$$

So that energy conservation for the reciprocal situation means 


$$
\sum_{\mu=s, p} \sum_{k=1} \eta_{(l ; \nu) \rightarrow(k ; \mu)}^{\prime}=\sum_{\mu=s, p} \sum_{k=1}^{N_{\max }} \eta_{(k ; \mu) \rightarrow(l ; \nu)}=1 .
$$

Superscripts $d$ and $u$ distinguish now between waves inside the cell that travel downward and upward, respectively. In the downward direction, under circular polarization the $(1 ; s)$ and $(1 ; p)$ modes carry half the irradiance from the external source, $P_{i} / 2$; the front internal reflectance (the reflectance of the upward wave when leaving the solar cell) for these modes must be low because it is the reverse path for the illumination from the sun, and it will be assumed 0. On the contrary, the front internal reflectance for the remaining $l$ $\neq 1$ orders is assumed to be 1 because, by the grating design, the total internal reflection takes place, while no radiation comes down from outside. Hence, in the absence of volume absorption, the following relationships apply at the front face:

$$
\begin{aligned}
& P_{(1 ; \mu)}^{d}=P_{i} / 2(\mu=s, p), \\
& P_{(k ; \mu)}^{d}=P_{(k ; \mu)}^{u}(k>1 ; \mu=s, p) .
\end{aligned}
$$

The downward modes when impinging on the grating excite the upward modes according to

$$
P_{(k ; \mu)}^{u}=\sum_{\nu=s, p} \sum_{l=1}^{N_{\max }} \eta_{(l ; \nu) \rightarrow(k ; \mu)} P_{(l ; \nu)}^{d} .
$$

On account of Eq. (20), Eq. (21) is as written

$$
P_{(k ; \mu)}^{u}-\sum_{\nu=s, p} \sum_{l=2}^{N_{\max }} \eta_{(l ; \nu) \rightarrow(k ; \mu)} P_{(l ; \nu)}^{u}=\sum_{\nu=s, p} \eta_{(1 ; \nu) \rightarrow(k ; \mu)} P_{i} / 2 .
$$

This is a set of linear equations (two for every excited mode) with the power carried by the modes as unknowns. It is trivial to verify that, if Eq. (19) applies, the result is

$$
P_{(k ; \mu)}^{u}=P_{i} / 2 \text {. }
$$

All modes carry the same power, irrespective of the diffraction efficiencies (provided none is exactly zero).

\section{B. Path length enhancement}

Following Eq. (A5) in the Appendix, with a perfectly reflecting grating at the back the average energy density can be calculated as the sum over the excited modes, each one carrying the same irradiance $P_{i} / 2$,

$$
w=\frac{2}{c} \sum_{l, \mu} \frac{P_{(l ; \mu)}}{\cos \left(\theta_{l}\right)}=\frac{2}{c} \sum_{l=1}^{N_{\max }} \frac{P_{i}}{\cos \left(\theta_{l}\right)} .
$$

The factor of 2 accounts for up- and down-traveling modes.

We shall consider now that the solar cell is illuminated by a set of plane waves that were individually considered in Sec. II. The electric field and the magnetic field are the sum of a large number of components in the incoming illumination cone. The coherence properties of sunlight can be altered by the optical system. As shown in the Appendix, if these components are not coherent the collective Poynting vector and energy density are locally the sum of the individual ones.

For extended illumination, the infinitesimal power carried in a given direction is $R d \Omega$, with $R$ the radiance (W $\mathrm{cm}^{-2} \mathrm{~s} \mathrm{rad}^{-1}$ ) and $d \Omega$ the differential of solid angle. This substitutes for the average Poynting vector in Eq. (15) so that Eq. (24) reads

$$
d w=\frac{R d \Omega}{c} .
$$

Let $(p, q, r)$ be the direction cosines of a wave vector; $p$ $=\sin (\theta) \cos (\phi)=\boldsymbol{u}_{x} \cdot \boldsymbol{u}_{r}, \quad q=\sin (\theta) \sin (\phi)=\boldsymbol{u}_{y} \cdot \boldsymbol{u}_{r}, \quad$ and $\quad r$ $=\cos (\theta)=\boldsymbol{u}_{z} \cdot \boldsymbol{u}_{r}$. In addition,

$$
\begin{aligned}
d p d q & =[\partial(p, q) / \partial(\theta, \phi)] d \theta d \phi=\sin \theta \cos \theta d \theta d \phi \\
& =\cos \theta d \Omega .
\end{aligned}
$$

By slightly varying the direction of the incident wave (subindices $i$ ) in Eq. (13), the following is obtained for the diffracted wave (subindices $r$ ):

$$
d \Omega_{i} \cos \theta_{i}=d p_{i} d q_{i}=d p_{r} d q_{r}=d \Omega_{r} \cos \theta_{r} .
$$

That proves that the étendue $(d p d q)$ conservation $^{14}$ is applicable to diffraction.

From Eq. (23) and remembering that $R d \Omega \cos \theta=P$, it is concluded that the radiance in lobe $l$ is the same as in the illumination bundle,

$$
R_{l}=R_{i}
$$

The sum over both polarization states is included in the previous formula. The energy density due to the bundle $l$ of diffracted rays is

$$
w_{l}=\frac{2}{c} \int_{\Omega_{l}} \int R_{l} d \Omega_{l} .
$$

The integral extends to the solid angle $\Omega_{l}$ of the diffracted lobe. Making use of Eq. (28) and considering that $R_{i}$ is constant in the incoming bundle, we have

$$
\begin{aligned}
w_{l} & =\frac{2 R_{i}}{c} \int_{l} \int_{l} \frac{d p d q}{\sqrt{1-p^{2}-q^{2}}} \Rightarrow w \\
& =\sum_{l=1}^{N_{\max }} w_{l}=\sum_{l=1}^{N_{\max }} \frac{2 R_{i}}{c} \int_{l} \int_{l} \frac{d p d q}{\sqrt{1-p^{2}-q^{2}}} .
\end{aligned}
$$

Extended to the region of the $p, q$ plane where the diffraction directions are found (i.e., where $\left|\boldsymbol{u}_{r t}\right| \leq 1$ ). w stands for the total energy density.

Light confinement is usually quantified by the path length increment, defined as the average length of the rays inside the cell $\bar{L}$ relative to the thickness $W$. It is found that ${ }^{16}$

$$
C_{I} \equiv \frac{\bar{L}}{W}=\frac{w c}{P_{i}},
$$

where $w$ is the average energy density, $c$ the speed of light in the medium, and $P_{i}$ the illumination irradiance. From Eq. (30) and the illumination irradiance $P_{i}=R_{i} \int \cos \theta \sin \theta d \theta d \phi$ $=R_{i} \pi \sin \left(\Theta_{x}\right)^{2}$, we obtain 


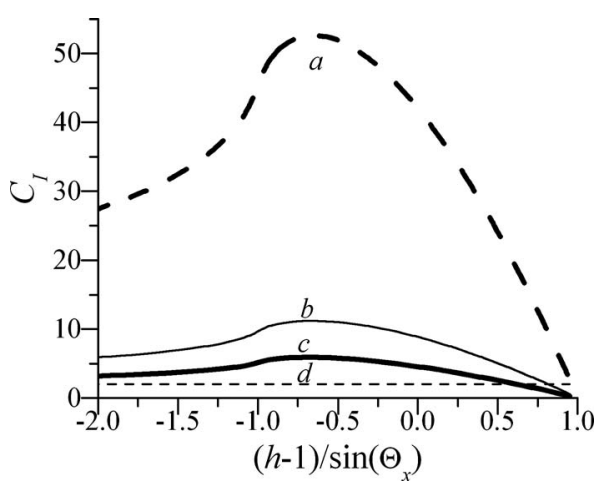

FIG. 10. Path enhancement factor for a single lobe for different illumination conditions as a function of $h-1 . h=1$ corresponds to normal illumination being diffracted horizontally. (a) direct sun illumination; (b) 500X ideal concentrator; (c) 500X, $1^{\circ}$ acceptance concentrator; (d) for $h=0$, vertical diffraction, $C_{I 0} \cong 2$.

$$
C_{I}=\frac{\sum_{l=1}^{N_{\max }} 2 \int_{l} \int \frac{d p d q}{\sqrt{1-p^{2}-q^{2}}}}{\pi \sin \left(\Theta_{x}\right)^{2}} .
$$

It is apparent that the more lobes are diffracted with equal tilt, the higher the path enhancement, so that a $2 \mathrm{D}$ grating can perform better than a $1 \mathrm{D}$ grating.

If no grating is placed at the back, but a planar perfect mirror instead, we would have only the $l=1$ lobe,

$$
C_{I 0}=\frac{4\left[1-\cos \left(\theta_{x}\right)\right]}{\sin \left(\Theta_{x}\right)^{2}} .
$$

As defined in Sec. II C, we denote by $h$ the tangential component of the diffraction direction for normal incidence, $h$ $=u_{r t}\left(\theta_{i}=0\right)$. The result of evaluating $C_{I}$ for the $\Theta_{x}$ values in Table I is given in Fig. 10. There is a maximum for $h=1$ $-0.655 \sin \left(\Theta_{x}\right) \cdot C_{I 0}$, for those thin beams, is approximately 2 as it corresponds to a double pass across the wafer. In case more than one order is diffracted the values of $C_{I}$ should be added as seen in Eq. (32).

\section{DISCUSSION AND CONCLUSIONS}

The infinite value involved for horizontal scattering in Eq. (1) has been the origin of overoptimistic statements concerning the potential of diffraction schemes for light confinement. Once the extended nature of the bundles used in solar energy is recognized, they are seen to be subjected to the general limits to light confinement.

The case considered here is a special one in that the diffracted lobes other than the zeroth order are completely confined by total internal reflection at the front, and the lobes do not overlap each other. In other cases, Eq. (23) would not be fulfilled and the energy density would be lower, decreasing the confinement.

From our analysis it can be concluded that the confinement is better if the diffracted lobes are more tilted and if more lobes are diffracted so that more area in the projected unit sphere is covered, hence favoring 2D versus 1D line gratings. This may enter into conflict with the requirements explained in the preceding paragraph. Besides, conditions vary with wavelength: as this is decreased, the tilt of dif- fracted rays changes (with the effect that can be appreciated from Fig. 10 and commented later on), more lobes and more closely packed are produced, but escaping rays may be excited.

For very short wavelength (with respect to the grating pitch) diffraction would proceed along all directions and the Lambertian case $^{7}$ is predicted (as in geometrical optics). Note that Lambertian structures using surfaces engraved with large motifs are widely used in the industry, for instance, anisotropically etched $(1,0,0)$ crystals follow accurately the Lambertian behavior. ${ }^{17,18}$

According to this concept the theoretical limit of confinement is $C_{I}=4 n^{2}$, independently of wavelength and the illumination bundle (assuming all directions within the critical angle are allowed to escape). For the value used of $n$ $=\sqrt{ } 11$, this gives $C_{I}=44$. Under the more realistic condition of having a dielectric at the back face, of index of refraction $n_{\text {optics }}=1.5$, and an absorber at the end of it, we would get $C_{I}=4 n^{2} /\left(1+n_{\text {optics }}^{2}\right)=13.5$. As we shall see below, this is less than what can be obtained with a nonconcentrator cell tracking the sun, but in this case no tracking is required and the result is independent of the wavelength.

With a back diffraction grating, from Fig. 10 it can be appreciated that, with six lobes at optimum tilt (plus the zero order), we would get $C_{I} \cong 320$ under direct sun illumination and $C_{I} \cong 40$ with an easy-to-make concentrator. Unlike the Lambertian approach, here the dependence with the wavelength is important. For a given grating, changes of wavelength lead to changes of $h$ by the same relative amount. From Fig. 10, we see that, to get $90 \%$ of the maximum $C_{I}$ value, $h$, and therefore $\lambda$, should be within $10 \%$ of the optimum for the $1^{\circ}$ acceptance angle concentrator, $3 \%$ for the ideal concentrator, and $0.1 \%$ for direct sun. These results would indicate that intensification by diffraction is a narrowband phenomenon. Note, however, that if lobes with different tilts are present the figures will be different since some lobes will work away from the optimum $h$ value.

The advantage that in this study can be achieved by diffraction confinement against randomized, Lambertian approach, at least for certain wavelength ranges, is due to the fact that the excitation of escaping rays outside the illumination cone but within the critical angle can be avoided in the former case, while it cannot in the latter one. The improvement relies on the limited angular extent of illumination bundles: for isotropic illumination, Lambertian surfaces achieve the upper limit of confinement and any further enhancement is forbidden by the second law of thermodynamics.

Asymmetrical grating lines have been proposed to be better than symmetrical ones because of reduced coupling to the zero order, escaping lobe after reflection at the front. ${ }^{19}$ The approximate character of our analysis makes the results independent of the shape of the diffracting elements and is not able to reveal these advantages, that will perhaps show up when absorption and reflection losses are included.

To obtain the results above, our paper uses a methodology widely used for the study of arrays of coherently radiating objects. We think that it is presented in a synthetic and general way for application to diffracting elements and al- 
lows understanding easily the effect of the changes of the illuminating wave in the 3D case, as well as handling noncoherent bundles of rays that will drastically limit the advantages of the horizontal scattering.

\section{ACKNOWLEDGMENTS}

This work is supported by the European Union Sixth Framework Program FULLSPECTRUM (Grant No. SESCT-2003-502620), the Spanish Education Ministry through the Plan Nacional-Consolider project GENESIS-FV (Grant No. CSD-2006-0004), and the Comunidad de Madrid project NUMANCIA (Grant No. S-05050/ENE/0310).

\section{APPENDIX}

Inside the cell volume, for a given wavelength, the field consists in a summation of plane waves,

$$
\boldsymbol{E}=\sum_{m} \boldsymbol{E}_{0 m} \exp \left(i \boldsymbol{k}_{m} \cdot \boldsymbol{r}\right), \quad \boldsymbol{H}=\sum_{m} \boldsymbol{H}_{0 m} \exp \left(i \boldsymbol{k}_{m} \cdot \boldsymbol{r}\right),
$$

$\boldsymbol{k}_{m}, \boldsymbol{E}_{0 m}$, and $\boldsymbol{H}_{0 m}$ fulfill the relationships (4). Waves inside the cell may exhibit different degrees of coherence. Diffracted orders at the surface grating will be highly coherent among themselves and with the incident wave, but waves coming from different illumination directions, or after reflection at another face if the substrate is thick, will not be correlated.

The average Poynting vector is obtained as

$$
\begin{aligned}
\langle\boldsymbol{S}(\boldsymbol{r})\rangle= & \frac{1}{2} \operatorname{Re}\left[\boldsymbol{E} \times \boldsymbol{H}^{*}\right] \\
= & \sum_{m}\left\langle\boldsymbol{S}_{m}\right\rangle+\frac{1}{2} \sum_{n \neq m} \sum_{m} \operatorname{Re}\left[\boldsymbol{E}_{0 m}\right. \\
& \left.\quad \times \boldsymbol{H}_{0 n}^{*} \exp \left(i\left(\boldsymbol{k}_{m}-\boldsymbol{k}_{n}\right) \cdot \boldsymbol{r}\right)\right] \\
= & \sum_{m} \frac{\left|\boldsymbol{E}_{0 m}\right|^{2}}{2 \eta} \frac{\boldsymbol{k}_{m}}{k}+\sum_{n \neq m} \sum_{m}\left(V _ { m n x } \boldsymbol { u } _ { x } \operatorname { c o s } \left(\left(\boldsymbol{k}_{m}-\boldsymbol{k}_{n}\right) \cdot \boldsymbol{r}\right.\right. \\
& \left.+\phi_{m n x}\right)+V_{m n y} \boldsymbol{u}_{y} \cos \left(\left(\boldsymbol{k}_{m}-\boldsymbol{k}_{n}\right) \cdot \boldsymbol{r}+\phi_{m n y}\right) \\
& \left.+V_{m n z} \boldsymbol{u}_{z} \cos \left(\left(\boldsymbol{k}_{m}-\boldsymbol{k}_{n}\right) \cdot \boldsymbol{r}+\boldsymbol{\phi}_{m n z}\right)\right),
\end{aligned}
$$

where $\left\langle\boldsymbol{S}_{m}\right\rangle$ pertains to a single wave and is spatially uniform. For $m \neq n$, interference terms arise that produce a spatially modulated vector with sinusoidal variation in the direction $\boldsymbol{k}_{m}-\boldsymbol{k}_{n} . V_{m n x}$ and $\phi_{m n x}$ are defined as the amplitude and phase of the $x$ component of the $m n$ interference term. If the interfering waves are not coherent, the random phase destroys interference, so that only single-wave Poynting vectors survive. However, interference among diffracted orders with high mutual coherence must be retained.

The average power flux $P$ per unit area through an arbitrary surface with normal vector $\boldsymbol{u}$ is calculated as

$$
P=\lim _{A \rightarrow \infty} \frac{1}{A} \int_{A} \int \boldsymbol{u} \cdot\langle\boldsymbol{S}\rangle d A=\sum_{m} \boldsymbol{u} \cdot\left\langle\boldsymbol{S}_{m}\right\rangle=\sum_{m} P_{m} .
$$

Given the oscillating nature of interference Poynting vectors, their contribution to net flux disappears. This justifies the treatment given to diffracted power flux in Sec. III A.

Similar considerations apply to the energy density. The local electromagnetic density $w_{\text {loc }}$ for a set of plane waves depends on position,

$$
\begin{aligned}
& \begin{aligned}
& w_{\mathrm{loc}}(\boldsymbol{r})=\frac{1}{4}\left(\varepsilon \boldsymbol{E} \cdot \boldsymbol{E}^{*}+\mu \boldsymbol{H} \cdot \boldsymbol{H}^{*}\right) \\
&=\sum_{m} w_{m}+\sum_{n<m} \sum_{m} C_{m n} \cos \left[\left(\boldsymbol{k}_{m}-\boldsymbol{k}_{n}\right) \cdot \boldsymbol{r}+\psi_{m n}\right], \\
& w_{m}=\frac{\varepsilon\left|\boldsymbol{E}_{0 m}\right|^{2}}{2}=\frac{\left\langle S_{m}\right\rangle}{c},
\end{aligned}
\end{aligned}
$$

where $w_{m}$ for a single plane wave is uniform. The interference terms show the same spatial modulation as the Poynting vector components and are written as cosine functions with $C_{m n}$ amplitude and $\psi_{m n}$ phase at the origin. Again, uncoherence translates into random phase terms that suppress interference.

The average electromagnetic density $w$ considered in Sec. III B is defined as

$$
w=\lim _{V \rightarrow \infty} \frac{1}{V} \int_{V} \iint w_{\mathrm{loc}}(\boldsymbol{r}) d V=\sum_{m} w_{m} .
$$

So that crossed terms disappear on spatial averaging.

${ }^{1}$ A. Luque and A. Martí, Phys. Rev. Lett. 78, 5014 (1997).

${ }^{2}$ A. Luque, A. Martí, C. Stanley, N. López, L. Cuadra, D. Zhou, J. L. Pearson, and A. McKee, J. Appl. Phys. 96, 903 (2004).

${ }^{3}$ P. Wahnón and C. Tablero, Phys. Rev. B 65, 165115 (2002).

${ }^{4}$ K. M. Yu, W. Walukievicz, J. Wu, W. Shan, J. W. Beeman, M. A. Scarpulla, O. D. Dubon, and P. Becla, Phys. Rev. Lett. 91, 246403 (2003).

${ }^{5}$ A. Luque, A. Martí, E. Antolín, and C. Tablero, Physica B (Amsterdam) 382, 320 (2006).

${ }^{6}$ A. Goezberger, Proceedings of the 15th IEEE Photovoltaic Specialists Conference, Kissimmee, FL, 1981 (unpublished), pp. 867-870.

${ }^{7}$ E. Yablonovitch and G. D. Cody, IEEE Trans. Electron Devices 29, 300 (1982).

${ }^{8}$ R. Morf and H. Kiess, Proceedings of the Ninth Photovoltaic Solar Energy Conference, Freiburg, 1989 (unpublished), pp. 313-315.

${ }^{9}$ M. Born and E. Wolf, Principles of Optics (Pergamon, Oxford, 1975).

${ }^{10} \mathrm{R}$. Petit, Electromagnetic Theory of Gratings (Springer-Verlag, Berlin, 1980).

${ }^{11} \mathrm{~F}$. Llopis, Estudio de la propiedades ópticas de células solares con microtextura, Universidad de La Laguna, 2004.

${ }^{12}$ O. J. F. Martin, C. Girard, and A. Dereux, Phys. Rev. Lett. 74, 526(1995).

${ }^{13}$ J. D. Jackson, Classical Electrodynamics (Wiley, New York, 1999).

${ }^{14} \mathrm{R}$. Winston and W. T. Welford, Optics of Non Imaging Concentrators (Academic, New York, 1979).

${ }^{15}$ N. A. Gippius, S. G. Tikhodeev, and T. Ishihara, Phys. Rev. B 72, 045138 (2005).

${ }^{16}$ J. C. Miñano, in Physical Limitations to Photovoltaic Energy Conversion, edited by A. Luque and G. L. Araujo (IOP, Bristol, 1990).

${ }^{17}$ I. Tobías, J. M. Rodríguez, and A. Luque, Prog. Photovoltaics 3, 177 (1995).

${ }^{18}$ J. M. Rodríguez, I. Tobías, and A. Luque, Sol. Energy Mater. Sol. Cells 45, 241 (1997)

${ }^{19}$ C. Heine and R. H. Morf, Appl. Opt. 34, 2476 (1995). 